QMW-PH-95-9

hep-th/9503021

March 2nd, 1995

\title{
Conformal Points and Duality of Non-Abelian Thirring Models and Interacting WZNW Models
}

\author{
Chris Hull and Oleg A. Soloviev ti \\ Physics Department, Queen Mary and Westfield College, \\ Mile End Road, London E1 4NS, United Kingdom
}

\begin{abstract}
We show that the strong coupling phase of the non-Abelian Thirring model is dual to the weak-coupling phase of a system of two WZNW models coupled to each other through a current-current interaction. This latter system is integrable and is related to a perturbed conformal field theory which, in the large $|k|$ limit, has a nontrivial zero of the perturbation-parameter beta-function. The non-Abelian Thirring model reduces to a free fermion theory plus a topological field theory at this critical point, which should therefore be identified with the isoscalar Dashen-Frishman conformal point. The relationship with the Gross-Neveu model is discussed.
\end{abstract}

March 1995

*e-mail: C.M.Hull@QMW.AC.UK

†e-mail: O.A.Soloviev@QMW.AC.UK 


\section{Introduction}

Gauged WZNW models [1], [2] provide a Lagrangian description of the GKO coset construction and hence of the minimal conformal models describing many statistical systems [3], [4], and of many exact string backgrounds [5]-[7]. These examples indicate the importance of Lagrangian CFT's formulated in terms of gauged WZNW models. An important observation is that gauged WZNW models can be understood in terms of two ordinary WZNW theories, with Kac-Moody currents $J_{1}, \bar{J}_{1}$ and $J_{2}, \bar{J}_{2}$ respectively, [8]-[10] coupled to each other through the isoscalar Thirring-like current-current interaction $\lambda J_{1}^{a} \bar{J}_{2}^{a}$ at a particular value of the Thirring coupling constant $\lambda$ [11]. However, this isoscalar coupling can be generalised to renormalizable current-current interactions of the form $S_{a b} J_{1}^{a} \bar{J}_{2}^{b}$ where $S_{a b}$ is a constant matrix and this raises the possibility that there may be other more general Thirring current-current interactions that give rise to new CFT's at special values of the Thirring coupling constants $S_{a b}$. The fact that WZNW models with completely general current-current interactions emerge naturally within the coadjoint orbit method [12] as well as in the theory of chiral WZNW models [13]- [15] give further hints that this might be the case. Interacting WZNW models arise also in the non-abelian bosonization of ordinary fermionic non-Abelian Thirring models with a generic current-current interaction [1], [16]-20].

Recently, one of us has discussed the conformal symmetry of non-Abelian Thirring models at the so-called Dashen-Frishman conformal points [20], 221] which are generalizations of the isoscalar nonperturbative conformal point discovered by Dashen and Frishman [22]. The conformal symmetry at the Dashen-Frishman conformal points can be demonstrated within the Hamiltonian current-current formalism [22], 21]. At the same time an attempt to associate these conformal points with zeros of the beta functions was not successful. It was suggested in [23] that the isoscalar Dashen-Frishman conformal point is related to the strong-coupling phase of the theory, but no computations were carried out. For example, one problem in establishing this was pointed out a long time ago by Gross and Neveu (see footnote in [24]). At the classical level the isoscalar Thirring model is equivalent to the Gross-Neveu theory after a Fierz rearrangement. A puzzle is that Gross 
and Neveu have shown that their model does not allow nontrivial conformal points to exist, while Dashen and Frishman have exhibited that their model does have a nontrivial conformal point. This suggests that the Gross-Neveu and the Dashen-Frishman models are different quantizations of the same classical model. However, it is possible that the Gross-Neveu and the Dashen-Frishman models may describe different phases of the same Thirring model, a weak coupling phase and a strong coupling phase respectively. Indeed, Fierz transformations which are allowed in the Gross-Neveu phase may be prohibited at the Dashen-Frishman conformal points. At these points different four fermionic combinations have different conformal dimensions, so that they cannot be manipulated using Fierz transformations. One of the aims of this paper is to investigate this possibility further.

Recently, the isoscalar conformal points of the non-Abelian Thirring model were found by the perturbative method in the large $N$ limit, where $N$ is the number of flavours. However, the method employed in [25] does not appeal to beta functions but rather to current algebra. In the present paper, we will investigate the conformal invariance of the Thirring model considered in [25] by studying the renormalization group flows.

We will show that the beta function approach leads to find nontrivial critical points of the fermionic Thirring model. "Bosonizing" the non-abelian Thirring model gives free fermions plus a system of two interacting WZNW models at negative level $k$. We will show that this interacting WZNW model in turn is related to a certain perturbed CFT which was introduced in [26], 27] (see also [28]) and shown to have a nontrivial infra-red conformal point. Thus the system of interacting WZNW models and the Thirring model also have such a fixed point, and in the fermionic Thirring model this conformal point will be argued to correspond to a free fermion phase, and so is a generalisation of the Dashen-Frishman isoscalar conformal point. Although the WZNW model at negative level has negative norm states and so is non-unitary, we will be interested in a subsector of a unitary theory. We will see that at the critical point the system of two interacting WZNW models at negative level becomes the bosonic part of a topological field theory in the large $|k|$ limit, so that the negative-norm states can be removed by introducing ghosts and restricting physical states to be those in the cohomology classes of the topological field theory BRST operator. 
The paper is organized as follows. In section 2, two interacting level $k$ WZNW models are introduced and in section 3 their action is re-expressed in a factorized form which will be convenient for implementing the $1 / k$ method. In section 4 we will consider a perturbation of a WZNW model by a relevant operator that is intimately related to the models of sections 2,3 . In section 5 we will discuss the conformal symmetry of the fermionic Thirring model at the conformal point found for the bosonic system. We conclude with some comments on the results obtained.

\section{Interacting WZNW models}

Let $S_{W Z N W}\left(g_{1}, k_{1}\right)$ and $S_{W Z N W}\left(g_{2}, k_{2}\right)$ be the actions of two WZNW models of levels $k_{1}$, $k_{2}$ and with $g_{1}, g_{2}$ taking values in the groups $G_{1}, G_{2}$ respectively:

$$
\begin{aligned}
& S_{W Z N W}\left(g_{1}, k_{1}\right)=\frac{-k_{1}}{4 \pi}\left\{\int \operatorname{Tr}\left|g_{1}^{-1} \mathrm{~d} g_{1}\right|^{2}+\frac{i}{3} \int \mathrm{d}^{-1} \operatorname{Tr}\left(g_{1}^{-1} \mathrm{~d} g_{1}\right)^{3}\right\}, \\
& S_{W Z N W}\left(g_{2}, k_{2}\right)=\frac{-k_{2}}{4 \pi}\left\{\int \operatorname{Tr}\left|g_{2}^{-1} \mathrm{~d} g_{2}\right|^{2}+\frac{i}{3} \int \mathrm{d}^{-1} \operatorname{Tr}\left(g_{2}^{-1} \mathrm{~d} g_{2}\right)^{3}\right\} .
\end{aligned}
$$

We shall add the following interaction term

$$
S_{I}=\frac{-k_{1} k_{2}}{\pi} \int d^{2} z \operatorname{Tr}^{2}\left(g_{1}^{-1} \partial g_{1} S \bar{\partial} g_{2} g_{2}^{-1}\right)
$$

to obtain用

$$
S\left(g_{1}, g_{2}, S\right)=S_{W Z N W}\left(g_{1}, k_{1}\right)+S_{W Z N W}\left(g_{2}, k_{2}\right)+S_{I}\left(g_{1}, g_{2}, S\right)
$$

with the coupling $S$ belonging to the direct product of two Lie algebras $\mathcal{G}_{1} \otimes \mathcal{G}_{2}$. The symbol $\operatorname{Tr}^{2}$ indicates a double tracing over the indices of a matrix from the tensor product $\mathcal{G}_{1} \otimes \mathcal{G}_{2}$. We shall assume that $S$ is invertible, so that $G_{1}$ and $G_{2}$ have the same dimension, and we shall later restrict ourselves to the case $G_{1}=G_{2}=G$.

The coupling matrix $S$ is dimensionless and the theory described by eq. (2.3) is conformally invariant classically. If $S=0$ and there is no interaction, the theory has $\hat{G}_{1}^{L} \times \hat{G}_{1}^{R} \times \hat{G}_{2}^{L} \times \hat{G}_{2}^{R}$ affine symmetry under which

$$
g_{1} \rightarrow \bar{\Omega}_{1}(\bar{z}) g_{1} \Omega_{1}(z), \quad g_{2} \rightarrow \bar{\Omega}_{2}(\bar{z}) g_{2} \Omega_{2}(z) .
$$

\footnotetext{
*A generalization to three and more interacting WZNW models is straightforward.
} 
The parameters $\Omega_{1,2}$ and $\bar{\Omega}_{1,2}$ are arbitrary independent Lie-group-valued functions of $z$ and $\bar{z}$ respectively. Remarkably, when $S \neq 0$, the interacting theory still has $\hat{G}_{1}^{L} \times \hat{G}_{1}^{R} \times$ $\hat{G}_{2}^{L} \times \hat{G}_{2}^{R}$ affine symmetry 29 , under which

$$
g_{1} \rightarrow \bar{\Omega}_{1}(\bar{z}) g_{1} h_{1} \Omega_{1}(z) h_{1}^{-1}, \quad g_{2} \rightarrow h_{2}^{-1} \bar{\Omega}_{2}(\bar{z}) h_{2} g_{2} \Omega_{2}(z)
$$

where $h_{1}, h_{2}$ are non-local functions of $g_{1}, g_{2}$ satisfying

$$
\bar{\partial} h_{1} h_{1}^{-1}=2 k_{2} \operatorname{Tr} S \bar{\partial} g_{2} g_{2}^{-1}, \quad h_{2}^{-1} \partial h_{2}=2 k_{1} \operatorname{Tr} S g_{1}^{-1} \partial g_{1}
$$

The $\bar{\Omega}_{1}$ and $\Omega_{2}$ transformations remain local, while the $\bar{\Omega}_{2}$ and $\Omega_{1}$ transformations are now intrinsically non-local, as they involve $h_{1}, h_{2}$.

The local $\bar{\Omega}_{1}$ and $\Omega_{2}$ symmetries are manifest, but the remaining non-local ones are by no means obvious, so we now give a direct proof of the $\Omega_{1}$ symmetry; an alternative way of understanding the symmetry is given in Appendix B. In infinitesimal form the $\Omega_{1}$ symmetry is given as follows

$$
g_{1} \rightarrow g_{1} \Lambda_{1}
$$

where

$$
\Lambda_{1}=h_{1} \Omega_{1} h_{1}^{-1}, \quad \Omega_{1}=1+\epsilon_{1}, \quad \bar{\partial} \epsilon_{1}=0 .
$$

The variation of the WZNW action for $g_{1}$ is

$$
\delta S_{W Z N W}\left(g_{1}\right)=-\frac{k_{1}}{2 \pi} \int d^{2} z \operatorname{Tr}\left(g_{1}^{-1} \partial g_{1} \bar{\partial} h_{1} h_{1}^{-1} \lambda_{1}-g_{1}^{-1} \partial g_{1} \lambda_{1} \bar{\partial} h_{1} h_{1}^{-1}\right)
$$

where

$$
\lambda_{1}=h_{1} \epsilon_{1} h_{1}^{-1}
$$

Further, using the definition of $h_{1}$ given by eq. (2.6) we obtain

$$
\delta S_{W Z N W}\left(g_{1}\right)=-\frac{k_{1} k_{2}}{\pi} \int d^{2} z \operatorname{Tr}^{2}\left(\lambda_{1} g_{1}^{-1} \partial g_{1} S \bar{\partial} g_{2} g_{2}^{-1}-g_{1}^{-1} \partial g_{1} \lambda_{1} S \bar{\partial} g_{2} g_{2}^{-1}\right)
$$

The variation of the interaction term (2.2) under (2.7), (2.8) is

$$
\delta S_{I}=-\frac{k_{1} k_{2}}{\pi} \int d^{2} z \operatorname{Tr}\left(-\lambda_{1} g_{1}^{-1} \partial g_{1} S \bar{\partial} g_{2} g_{2}^{-1}+g_{1}^{-1} \partial g_{1} \lambda_{1} S \bar{\partial} g_{2} g_{2}^{-1}+\partial \lambda_{1} S \bar{\partial} g_{2} g_{2}^{-1}\right)
$$

Adding together eq. (2.11) and eq. (2.12), we finally obtain

$$
\delta S\left(g_{1}, g_{2}, S\right)=-\frac{k_{1} k_{2}}{\pi} \int d^{2} z \operatorname{Tr}^{2} \partial \lambda_{1} S \bar{\partial} g_{2} g_{2}^{-1}
$$


Using (2.6), the expression on the right hand side of eq. (2.13) can be rewritten in terms of $h_{1}$ as follows

$$
\begin{aligned}
\delta S\left(g_{1}, g_{2}, S\right) & =-\frac{k_{1}}{2 \pi} \int d^{2} z \operatorname{Tr} \partial \lambda_{1} \bar{\partial} h_{1} h_{1}^{-1}=\frac{k_{1}}{2 \pi} \int d^{2} z \operatorname{Tr} \lambda_{1} \partial\left(\bar{\partial} h_{1} h_{1}^{-1}\right) \\
& =\frac{k_{1}}{2 \pi} \int d^{2} z \operatorname{Tr} \lambda_{1} h_{1} \bar{\partial}\left(h_{1}^{-1} \partial h_{1}\right) h_{1}^{-1}=-\frac{k_{1}}{2 \pi} \int d^{2} z \operatorname{Tr} \bar{\partial}\left(h_{1}^{-1} \lambda_{1} h_{1}\right) h_{1}^{-1} \partial h_{1}=0,
\end{aligned}
$$

because $h_{1}^{-1} \lambda_{1} h_{1}=\epsilon_{1}$, which is holomorphic (2.8). Thus the action (2.3) is invariant, up to a surface term, under $(2.7),(2.8)$, and the $\bar{\Omega}_{2}$ symmetry of the action in eq. (2.3) can be proven in the similar fashion.

The equations of motion of the theory (2.3) can be written as

$$
\partial \overline{\mathcal{J}}_{1}=0, \quad \bar{\partial} \mathcal{J}_{2}=0
$$

where

$$
\begin{aligned}
& \overline{\mathcal{J}}_{1}^{\bar{a}}=\bar{J}_{1}^{\bar{a}}+2 k_{2} \phi_{1}^{a \bar{a}} S^{a \bar{b}} \bar{J}_{2}^{\bar{b}}, \\
& \mathcal{J}_{2}^{a}=J_{2}^{a}+2 k_{1} \phi_{2}^{a \bar{a}} S^{b \bar{a}} J_{1}^{b} .
\end{aligned}
$$

and

$$
\begin{aligned}
J_{1}^{a} & =-\frac{k}{2} \operatorname{Tr}\left(g_{1}^{-1} \partial g_{1} t_{1}^{a}\right), \\
J_{2}^{a} & =-\frac{k}{2} \operatorname{Tr}\left(g_{2}^{-1} \partial g_{2} t_{2}^{a}\right), \\
\bar{J}_{1}^{\bar{a}} & =-\frac{k}{2} \operatorname{Tr}\left(\bar{\partial} g_{1} g_{1}^{-1} t_{1}^{\bar{a}}\right), \\
\bar{J}_{2}^{\bar{a}} & =-\frac{k}{2} \operatorname{Tr}\left(\bar{\partial} g_{2} g_{2}^{-1} t_{2}^{\bar{a}}\right), \\
\phi_{1}^{a \bar{a}} & =\operatorname{Tr}\left(g_{1} t_{1}^{a} g_{1}^{-1} t_{1}^{\bar{a}}\right), \\
\phi_{2}^{a \bar{a}} & =\operatorname{Tr}\left(g_{2} t_{2}^{a} g_{2}^{-1} t_{2}^{\bar{a}}\right),
\end{aligned}
$$

where $t_{1,2}^{a}$ are the generators of the Lie algebras $\mathcal{G}_{1,2}$ associated with the Lie groups $G_{1,2}$,

$$
\begin{aligned}
& {\left[t_{1}^{a}, t_{1}^{b}\right]=f_{(1) c}^{a b} t_{1}^{c},} \\
& {\left[t_{2}^{a}, t_{2}^{b}\right]=f_{(2) c}^{a b} t_{2}^{c},}
\end{aligned}
$$


with $f_{(1,2) c}^{a b}$ the structure constants. The local conserved currents $\overline{\mathcal{J}}_{1}, \mathcal{J}_{2}$ correspond to the local $\bar{\Omega}_{1}$ and $\Omega_{2}$ symmetries, and there are in addition two non-local conserved currents $\mathcal{J}_{1}, \overline{\mathcal{J}}_{2}$ corresponding to the non-local $\bar{\Omega}_{2}$ and $\Omega_{1}$ symmetries. These two local and two non-local conserved currents generate an infinite number of conserved charges that lead to the integrability of the system of two interacting WZNW models with arbitrary invertible coupling matrix $S$ (at least classically). The classical current algebras generated by $\mathcal{J}_{1}^{a}$ and $\overline{\mathcal{J}}_{1}^{\bar{a}}$ in eqs. (2.16) have levels $k_{2}$ and $k_{1}$ respectively, but we shall see in section 5 that there is a conformal point at which these become renormalized to $-k_{2}-2 c_{V}\left(G_{2}\right)$ and $-k_{1}-2 c_{V}\left(G_{1}\right)$ respectively. The current algebras can in principle be used to solve the model; we hope to return to this in the future, and here adopt a different strategy.

From now on, we shall take $G_{1}=G_{2}=G, k_{1}=k_{2}=k$. WZNW models are usually considered for compact groups and positive integral level. In this paper we shall also be interested in WZNW models at negative (integer) $k$ with compact $G$. The WZNW model on compact $G$ at negative level is a complicated system whose spectrum contains states of negative norm. We will discuss in section 5 an important situation in which nonunitary states of WZNW models at negative level decouple and can be dropped to leave a unitary theory.

The classical conformal invariance of the the system (2.3) will in general be spoiled by quantum conformal anomalies. This can be seen in the simple example in which $G=S U(2), k=1$ and $S=\lambda I$, where $I$ is the identity in $s u(2) \otimes s u(2)$. When $\lambda$ is small, the theory given by (2.3) is equivalent to the sine-Gordon model [31] which is not a conformal theory for general values of the coupling. However, as for the Sine-Gordon model, there can be special values of the coupling for which the conformal invariance extends to the quantum level. For example, if we take $\lambda=1 / 2 k$ so that $S=I / 2 k$, then using the Polyakov-Wiegmann formula [11] we obtain

$$
S\left(g_{1}, g_{2}, S=I / 2 k\right)=S_{W Z N W}(f, k),
$$

where $f=g_{1} g_{2}$. The conformal invariance of this theory follows immediately from the fact that the right hand side of eq. (2.19) is a conformal WZNW model. This Polyakov-Wiegmann conformal point has an extra gauge invariance under $g_{1} \rightarrow g_{1} \Lambda, g_{2} \rightarrow$ 
$\Lambda^{-1} g_{2}$, where $\Lambda$ is arbitrary group element. Quantization of the theory at the PolyakovWiegmann conformal point requires gauge fixing in the usual way. The gauge symmetry acts algebraically (without derivatives) on $g_{1}$ and $g_{2}$, so that with an algebraic choice such as $g_{2}=1$ there are no dynamical ghosts and the theory reduces to a conventional WZNW model. Thus (2.3) has at least one conformal point, and one of the aims of this paper will be to find others.

\section{Expansion in the coupling constants}

We shall consider coupling matrices $S$ of the form

$$
S=\sigma \cdot \hat{S}
$$

where $\hat{S}$ is some matrix and $\sigma$ is a small parameter. The interaction term in eq. (2.3) is linear in $\sigma$, but we will recast this theory in a new form without interaction between $g_{1}$ and $g_{2}$ but with a nonlinear dependence on $\sigma$ instead.

To this end, we make the following change of variables

$$
\begin{aligned}
& g_{1} \rightarrow \tilde{g}_{1}, \\
& g_{2} \rightarrow h\left(\tilde{g}_{1}\right) \cdot \tilde{g}_{2},
\end{aligned}
$$

where the function $h\left(\tilde{g}_{1}\right)$ is the solution of the following equation

$$
\partial h \cdot h^{-1}=-2 k \sigma \operatorname{Tr} \hat{S} \tilde{g}_{1}^{-1} \partial \tilde{g}_{1}
$$

This determines $h$ up to changes of the form

$$
h \rightarrow h \Lambda(\bar{z})
$$

where $\Lambda$ is an antiholomorphic matrix function, $\partial \Lambda=0$, and we will pick some particular solution $h_{0}(z, \bar{z})$.

Writing the action given by eq. (2.3) in terms of the new variables $\tilde{g}_{1}, \tilde{g}_{2}$ and using the Polyakov-Wiegmann formula, we obtain

$$
S\left(g_{1}, g_{2}, k\right) \rightarrow S\left(\tilde{g}_{1}, \tilde{g}_{2}, k\right)=S_{W Z N W}\left(\tilde{g}_{2}, k\right)+S_{W Z N W}\left(\tilde{g}_{1}, k\right)
$$




$$
+S_{W Z N W}\left(h_{0}, k\right)-\frac{k^{2} \sigma}{\pi} \int d^{2} z \operatorname{Tr}^{2} \tilde{g}_{1}^{-1} \partial \tilde{g}_{1} \hat{S} \bar{\partial} h_{0} h_{0}^{-1},
$$

where $h_{0}$ is a non-local function of $\tilde{g}_{1}$ satisfying (3.22). Remarkably, after this change of variables, the field $\tilde{g}_{2}$ completely decouples from $\tilde{g}_{1}$. Note that the Jacobian of the change of variables in eq. (3.21) is formally equal to one.

The price we pay for the factorization is a highly nonlocal theory for the variable $\tilde{g}_{1}$. While $\tilde{g}_{2}$ is governed simply by a WZNW action, the action for $\tilde{g}_{1}$ is

$S\left(\tilde{g}_{1}\right)=S_{W Z N W}\left(\tilde{g}_{1}, k\right)+S_{W Z N W}\left(h_{0}\left(\tilde{g}_{1}\right), k\right)-\frac{k^{2} \sigma}{\pi} \int d^{2} z \operatorname{Tr}^{2} \tilde{g}_{1}^{-1} \partial \tilde{g}_{1} \hat{S} \bar{\partial} h_{0}\left(\tilde{g}_{1}\right) h_{0}^{-1}\left(\tilde{g}_{1}\right)$,

which is nonlocal as $h_{0}$ is a non-local function of $\tilde{g}_{1}$. By considering $\bar{\partial}\left(\partial h \cdot h^{-1}\right)$ and using eq. (3.22), we obtain

$$
\begin{aligned}
& \bar{\partial} h_{0} h_{0}^{-1}(z, \bar{z})=-2 k \sigma \operatorname{Tr} \hat{S} \tilde{g}_{1}^{-1}(z, \bar{z}) \bar{\partial}_{\bar{z}} \tilde{g}_{1}(z, \bar{z}) \\
+ & 2 k \sigma \int d^{2} y \bar{\partial}_{\bar{z}} G(z, \bar{z} ; y, \bar{y}) v(y, \bar{y}),
\end{aligned}
$$

where

$$
\begin{aligned}
v(y, \bar{y}) & =\operatorname{Tr} \hat{S} \tilde{g}_{1}^{-1}(y, \bar{y})\left[\bar{\partial}_{\bar{y}} \tilde{g}_{1}(y, \bar{y}) \tilde{g}_{1}^{-1}(y, \bar{y}), \partial_{y} \tilde{g}_{1}(y, \bar{y}) \tilde{g}_{1}^{-1}(y, \bar{y})\right] \tilde{g}_{1}(y, \bar{y}) \\
& +\left[\operatorname{Tr} \hat{S} \tilde{g}_{1}^{-1}(y, \bar{y}) \partial_{y} \tilde{g}_{1}(y, \bar{y}), \bar{\partial} h_{0}(y, \bar{y}) h_{0}^{-1}(y, \bar{y})\right]
\end{aligned}
$$

and the Green function $G(z, \bar{z} ; y, \bar{y})$ satisfies

$$
\bar{\partial}_{\bar{z}} \partial_{z} G(z, \bar{z} ; y, \bar{y})=\delta(z, y) \delta(\bar{z}, \bar{y}) .
$$

We regularise the Green function in such a way that

$$
\lim _{y \rightarrow z, \bar{y} \rightarrow \bar{z}} \partial_{z} G(z, \bar{z} ; y, \bar{y})=0
$$

so that, despite its nonlocality, the right hand side of eq. (3.26) is well defined, even when $(y, \bar{y}) \rightarrow(z, \bar{z})$. Note that eq. (3.26) fixes the dependence of $h_{0}$ on $\bar{z}$.

In the Wess-Zumino term in $S_{W Z N W}\left(h_{0}\left(\tilde{g}_{1}\right), k\right), h_{0}$ and $\tilde{g}_{1}$ are extended to functions of $z, \bar{z}$ and an extra coordinate $t$ and an equation analogous to (3.26) can be found for $\partial_{t} h_{0} h_{0}^{-1}$. Then $h_{0}$ appears in (3.25) only through its derivatives, so that (3.25) can be 
written in terms of $\tilde{g}_{1}$ using (3.22), (3.26) and the analogous equation for $\partial_{t} h_{0} h_{0}^{-1}$. The resulting action includes a non-local term of order $\sigma^{2}$ of the form

$$
\frac{k \sigma^{2}}{\pi} \int d^{2} z \bar{\partial}\left(\tilde{g}_{1}^{-1} \partial \tilde{g}_{1}\right) \Psi
$$

where

$$
\Psi(z, \bar{z})=k^{2} \int d^{2} y G(z, \bar{z} ; y, \bar{y}) \operatorname{Tr} \hat{S} v(y, \bar{y})
$$

On making a further change of variables

$$
\tilde{g}_{1} \rightarrow \tilde{g}_{1}\left(1+2 \sigma^{2} \Psi+\mathcal{O}\left(\sigma^{3}\right)\right)
$$

we obtain an action which is local up to order $\sigma^{3}$ and which takes the simple form

$$
S\left(\tilde{g}_{1}\right)=S_{W Z N W}\left(\tilde{g}_{1}, k\right)+\frac{k^{3} \sigma^{2}}{\pi} \int d^{2} z \operatorname{Tr}\left(\operatorname{Tr} \hat{S} \tilde{g}_{1}^{-1} \partial \tilde{g}_{1} \cdot \operatorname{Tr} \hat{S} \tilde{g}_{1}^{-1} \bar{\partial} \tilde{g}_{1}\right)+\mathcal{O}\left(\sigma^{3}\right) .
$$

Further, eq. (3.32) can be rewritten as

$$
S\left(\tilde{g}_{1}\right)=S_{W Z N W}\left(\tilde{g}_{1}, k\right)-\tau \int d^{2} z \Sigma_{a b} \tilde{J}_{1}^{a} \overline{\tilde{J}}_{1}^{\bar{b}} \tilde{\phi}_{1}^{b \bar{b}}+\mathcal{O}\left(\sigma^{3}\right),
$$

where we have introduced the notation

$$
\tau=-\frac{4 k \sigma^{2}}{\pi}, \quad \Sigma_{a b}=\hat{S}^{a \bar{a}} \hat{S}^{b \bar{a}}
$$

The expression for $S\left(\tilde{g}_{1}\right)$ obtained in eq. (3.33) presents an expansion in the coupling $\sigma$ around the conformal theory described by the level $k$ WZNW model $S_{W Z N W}\left(\tilde{g}_{1}, k\right)$. In the next section we will show that the operator $\Sigma_{a b} \tilde{J}_{1}^{a} \tilde{\bar{J}}_{1}^{\bar{b}} \tilde{\phi}_{1}^{b \bar{b}}$ provides a relevant renormalizable perturbation of the WZNW model at negative level $k$.

\section{Relevant perturbations}

In this section we are going to discuss a certain perturbed CFT which will be shown to coincide with the theory given by eq. (3.33), to lowest order in the perturbation parameter.

Consider a WZNW model on group $G$ with level $l$. We define the following composite operator 26] - 28

$$
O^{L, \bar{L}}=L_{a b} \bar{L}_{\bar{a} \bar{b}}: J^{a} \bar{J}^{\bar{a}} \phi^{b \bar{b}}:
$$


where

$$
\begin{aligned}
J & =J^{a} t^{a}=-\frac{l}{2} g^{-1} \partial g, \\
\bar{J} & =\bar{J}^{a} t^{a}=-\frac{l}{2} \bar{\partial} g g^{-1}, \\
\phi^{a \bar{a}} & =\operatorname{Tr}: g^{-1} t^{a} g t^{\bar{a}}: .
\end{aligned}
$$

The product of the three operators in eq. (4.35) is defined by [26]

$$
O^{L, \bar{L}}(z, \bar{z})=L_{a b} \bar{L}_{\bar{a} \bar{b}} \oint \frac{d w}{2 \pi i} \oint \frac{d \bar{w}}{2 \pi i} \frac{J^{a}(w) \bar{J}^{\bar{a}}(\bar{w}) \phi^{b \bar{b}}(z, \bar{z})}{|z-w|^{2}}
$$

where the product in the numerator of the integrand is to be understood as an OPE. It is easy to see that the given product does not contain singular terms provided the matrices $L_{a b}$ and $\bar{L}_{\bar{a} \bar{b}}$ are symmetric [.

The operator $O^{L, \bar{L}}$ is a level one affine descendant of the affine primary field $\phi$. Indeed, $O^{L, \bar{L}}$ can be presented in the form

$$
O^{L, \bar{L}}(0)=L_{a b} \bar{L}_{\bar{a} \bar{b}} J_{-1}^{a} \bar{J}_{-1}^{\bar{a}} \phi^{b \bar{b}}(0)
$$

where

$$
J_{m}^{a}=\oint \frac{d w}{2 \pi i} w^{m} J^{a}(w), \quad \bar{J}_{m}^{a}=\oint \frac{d \bar{w}}{2 \pi i} \bar{w}^{m} \bar{J}^{a}(\bar{w}) .
$$

Being an affine descendant, the operator $O^{L, \bar{L}}$ is a Virasoro primary operator. Indeed, one can check that the state $O^{L, \bar{L}}(0)|0\rangle$ is a highest weight vector of the Virasoro algebra, with $|0\rangle$ the $S L(2, C)$-invariant vacuum. That is,

$$
L_{0} O^{L, \bar{L}}(0)|0\rangle=\Delta_{O} O^{L, \bar{L}}(0)|0\rangle, \quad L_{m>0} O^{L, \bar{L}}(0)|0\rangle=0 .
$$

Here $L_{n}$ are the generators of the Virasoro algebra associated with the affine-Sugawara stress-energy tensor of the conformal WZNW model. By using the Knizhnik-Zamolodchikov formula for anomalous conformal dimensions [10], it is straightforward to show that

$$
\Delta_{O}=\bar{\Delta}_{O}=1+\frac{c_{V}}{l+c_{V}}
$$

${ }^{\dagger}$ Indeed, the field $\phi$ is an affine primary vector. Therefore, its OPE with the affine current $J$ is

$$
J^{a}(w) \phi^{b \bar{b}}(z, \bar{z})=\frac{f^{a b c}}{(w-z)} \phi^{c \bar{b}}(z, \bar{z})+\text { regular terms } .
$$

Substituting this formula into eq. (4.37), one can see that only regular terms will contribute provided $L_{a b}$ is a symmetric matrix. 
Here $\bar{\Delta}_{O}$ is the conformal dimension of $O^{L, \bar{L}}$ associated with antiholomorphic conformal transformations. The quantity $c_{V}$ is defined by

$$
f_{d}^{a c} f_{c}^{b d}=-c_{V} g^{a b}
$$

with $g^{a b}$ the Killing metric.

From the formula (4.41) for anomalous conformal dimensions of the operator $O^{L, \bar{L}}$, it is clear that when

$$
l<-2 c_{V}
$$

the conformal dimensions are in the range between 0 and 1 . Hence, when condition (4.43) is fulfilled, the operator $O^{L, \bar{L}}$ becomes a relevant conformal operator. Correspondingly, for positive $l$ the operator $O^{L, \bar{L}}$ is irrelevant.

We are interested here in relevant operators, so we shall consider levels $l$ which are negative integers. The conformal WZNW model with negative level is a nonunitary theory because there are states of negative norm in its spectrum. Let us show that the operator $O^{L, \bar{L}}$ corresponds to a unitary highest weight representation of the Virasoro algebra of the nonunitary WZNW model. Indeed, as we have pointed out above, $O^{L, \bar{L}}$ has positive conformal dimensions. Besides, the condition (4.43) guarantees that the Virasoro central charge of the nonunitary WZNW model is greater than one,

$$
c_{W Z N W}(l)=\frac{l \operatorname{dim} G}{l+c_{V}}=\operatorname{dim} G+\mathcal{O}(1 / l)>1 .
$$

Thus, the operator $O^{L, \bar{L}}$ lies in the unitary range of the Kac-Kazhdan determinant and so it provides a unitary representation of the Virasoro algebra. Note that although $O^{L, \bar{L}}$ belongs to a nonunitary representation of the affine algebra, the Virasoro representation generated by $O^{L, \bar{L}}$ is unitary. This can be understood from the point of view of analytic continuation of nonunitary WZNW models. Indeed, one way of thinking about certain nonunitary WZNW models is to treat them as analytic continuations of unitary WZNW models based on compact groups to ones on noncompact groups. In the course of analytic continuation, the affine operators become nonhermitian, while the Virasoro operators continue to be hermitian. We do not insist on representations of the affine algebra since 
we are interested in scaling properties of the model. These properties allow us to consider the operator $O^{L, \bar{L}}$ as a physical conformal operatorf.

Let us turn to the fusion algebra of $O^{L, \bar{L}}$. Clearly, operators $O^{L, \bar{L}}$ with arbitrary symmetric matrices $L_{a b}, \bar{L}_{\bar{a} \bar{b}}$ are Virasoro primary vectors with the same conformal dimensions. However, their fusion algebras may be different. Among all such operators, there are operators which obey the following fusion algebra 28]

$$
O^{L, \bar{L}} \cdot O^{L, \bar{L}}=\left[O^{L, \bar{L}}\right]+[I]+\ldots,
$$

where the square brackets denote the contributions of $O^{L, \bar{L}}$ and the identity operator $I$ and their descendants, whereas the dots stand for all other operators with irrelevant conformal dimensions 3 . These operators must be singlets under $G$ which are constructed from $g$ and its derivatives. From the results of [10] on the spectrum of the WZNW model, it follows that for general $L$, the only relevant singlet operators that can appear on the right hand side of (4.45) are of the form $O^{\tilde{L}, \tilde{\bar{L}}}$ and $I$, for some symmetric matrices $\tilde{L}, \tilde{\bar{L}}$; this is shown in detail for $G=S U(2)$ in appendix C. As shown in 28], requiring that $L=\tilde{L}$ so that the fusion algebra is given by eq. (4.45) results in complicated algebraic equations for the matrices $L, \bar{L}$. In general, these equations may have many solutions each of which will yield an operator $O^{L, \bar{L}}$ satisfying the closed fusion algebra in eq. (4.45). In what follows, we will be dealing with the large $|l|$ limit. In this limit there are asymptotic solutions for $L_{a b}, \bar{L}_{\bar{a} \bar{b}} 28$

$$
\begin{aligned}
L_{a b} & =\frac{1}{\sqrt{c}_{V}} g_{a b}+\mathcal{O}(1 / l), \\
\bar{L}_{\bar{a} \bar{b}} & =\frac{1}{\sqrt{c}_{V}} g_{\bar{a} \bar{b}}+\mathcal{O}(1 / l) .
\end{aligned}
$$

Now it becomes clear that with the given values of the matrices $L, \bar{L}$ one can make use of the operator $O^{L, \bar{L}}$ to perform relevant renormalizable perturbations of the level $l$ WZNW model.

\footnotetext{
${ }^{\ddagger}$ In the next section we will show that interacting nonunitary WZNW models emerge as the bosonic subsector of a certain unitary theory. Adding a ghost sector gives a theory with a unitary physical subsector defined by the cohomology of a certain BRST operator.

$\S$ We emphasize that we are computing the OPE of conformal operators of the conformal WZNW model.
} 
The perturbed theory is defined as follows

$$
S(\epsilon)=S_{W Z N W}(g, l)-\epsilon \int d^{2} z O^{L, \bar{L}}(z, \bar{z})+\mathcal{O}\left(\epsilon^{2}\right)
$$

This theory has been proven [28] to possess a nontrivial infra-red conformal point at

$$
\epsilon=\epsilon^{*} \equiv-\frac{2 c_{V}}{\pi l}+\mathcal{O}\left(l^{-2}\right)
$$

for large $|l|$. In the evaluation of $\epsilon^{*}$, the expansion of the beta function in $\epsilon$ up to $\epsilon^{3}$ terms has been used [26], [27], and this is sufficient for large $|l|$. Substituting this value of $\epsilon$ gives the new CFT

$$
S\left(\epsilon^{*}\right)=S_{W Z N W}(g, l)-\epsilon^{*} \int d^{2} z O^{L, \bar{L}}(z, \bar{z})+\mathcal{O}\left(l^{-2}\right) .
$$

It was shown in [26] that this new CFT has, in the large $|l|$ limit, the same Virasoro central charge and that the operators have the same anomalous conformal dimensions as for the WZNW model with level - $l$. It was argued in [26] that the WZNW model at level $l$ flows to a model which, at the infra-red fixed point, is the WZNW model with level given approximately by $-l$ for large $|l|$. We will argue later that the exact result for the level of the new WZNW theory is $-l-2 c_{V}$.

It is interesting to compare the model just obtained with the theory discussed in the previous section. The model in eq. (4.49) coincides with the expression in eq. (3.33) provided $k=l$ and

$$
\sigma^{2}=\frac{c_{V}}{2 k^{2}}, \quad \hat{S}^{a \bar{a}} \hat{S}^{b \bar{a}}=\frac{g^{a b}}{c_{V}} .
$$

Thus when these conditions are satisfied, the system of two interacting WZNW models at level $k$ is conformally invariant. When $|k|$ is large, there are two solutions

$$
S_{ \pm}^{*}= \pm \frac{I}{\sqrt{2} k} .
$$

In fact there are further solutions in the large $|k|$ limit, which correspond to other solutions $L_{a b}$ of the algebra in eq. (4.45) 28]. However only the two solutions displayed above are nondegenerate, so that $\left(S^{*}\right)^{-1}$ exists [28]. The invertibility will be an important property in what follows. The status of the $S_{+}^{*}$ solution is unclear: in flowing from zero coupling to $S_{+}^{*}$, one passes through the Polyakov-Wiegmann conformal point $S_{P W}^{*}=I / 2 k$, at which 
there is some evidence that a phase transition occurs. This means that one might expect some non-analytic behaviour in continuing the coupling constant through the PolyakovWiegmann conformal point to $S_{+}^{*}$. However, it might be the case that the $S_{+}^{*}$ solution is related to the $S_{-}^{*}$ solution by some kind of duality transformation. In the next section, we shall investigate the second solution $S_{-}^{*}$ using a fermionic version of the theory.

\section{Free fermion phase of the non-Abelian Thirring model}

Interacting WZNW models at negative level naturally emerge in the course of the path integral bosonization of an ordinary non-Abelian fermionic Thirring model [11] and this is the main motivation for the study of the model (2.3). Consider the fermionic non-Abelian Thirring model described by the following action

$$
S_{F}=\frac{1}{4 \pi} \int d^{2} z\left(\bar{\psi}_{L} \bar{\partial} \psi_{L}+\bar{\psi}_{R} \partial \psi_{R}-G_{a \bar{a}} J_{L}^{a} J_{R}^{\bar{a}}\right)
$$

where $\psi_{L}$ and $\psi_{R}$ are complex Weyl spinors transforming as the fundamental representations of groups $G_{L}$ and $G_{R}$ respectively. The spinors $\psi_{R}^{i}$ and $\psi_{L}^{\bar{i}}$ carry flavour indices $i=1, \ldots, k_{R}$ and $\bar{i}=1, \ldots, k_{L}$. The last term in eq. (5.52) describes the general interaction between fermionic currents

$$
J_{L}^{a}=\bar{\psi}_{L} t^{a} \psi_{L}, \quad J_{R}^{\bar{a}}=\bar{\psi}_{R} t^{\bar{a}} \psi_{R}
$$

Here $t^{a}, t^{\bar{a}}$ are the generators in the Lie algebras $\mathcal{G}_{L}, \mathcal{G}_{R}$ :

$$
\left[t^{a}, t^{b}\right]=f_{c}^{a b} t^{c}, \quad\left[t^{\bar{a}}, t^{\bar{b}}\right]=f_{\bar{c}}^{\bar{a} \bar{b}} t^{\bar{c}}
$$

$G_{a \bar{a}}$ is a coupling constant matrix. In what follows we will suppose $G_{L}=G_{R}=G, k_{L}=$ $k_{R}=N$.

The action (5.52) can be rewritten in the equivalent form

$$
\tilde{S}_{F}=\frac{1}{4 \pi} \int d^{2} z\left(\bar{\psi}_{L} \bar{\partial} \psi_{L}+\bar{\psi}_{R} \partial \psi_{R}+A_{a} J_{L}^{a}+\bar{A}_{\bar{a}} J_{R}^{\bar{a}}+\left(G^{-1}\right)^{a \bar{a}} A_{a} \bar{A}_{\bar{a}}\right),
$$


where $A_{a}, \bar{A}_{\bar{a}}$ are auxiliary vector fields. This yields the fermionic Thirring model after eliminating the fields $A, \bar{A}$ by using their algebraic equations of motion. After introducing these auxiliary fields, the action (5.55) takes a Gaussian form for the fermions.

Note that the fields $A, \bar{A}$ are not to be treated as gauge vector fields. The theory is only gauge invariant if $G^{-1} \sim \hbar I$. In particular, for general $G$ there is no gauge symmetry that can be used to fix local counterterms. The partition functions $Z_{F}(G)$ and $\tilde{Z}_{F}$ derived from the two forms of the action, (5.52) and (5.55), are related by

$$
Z_{F}(G)=J^{-1} \tilde{Z}_{F}
$$

where

$$
\begin{aligned}
Z_{F}(G) & =\int \mathcal{D} \psi_{L} \mathcal{D} \psi_{R} \mathrm{e}^{-S_{F}} \\
\tilde{Z}_{F} & =\int \mathcal{D} \psi_{L} \mathcal{D} \psi_{R} \mathcal{D} A \mathcal{D} \bar{A} \mathrm{e}^{-\tilde{S}_{F}} \\
J & =\int \mathcal{D} A \mathcal{D} \bar{A} \mathrm{e}^{-\frac{1}{4 \pi} \int d^{2} z A G^{-1} \bar{A}} .
\end{aligned}
$$

The quantity $J$ is an inessential constant which we will drop (see Appendix A). The functional integrals over the spinor fields are Gaussian and the variables $\psi_{L}$ and $\psi_{R}$ do not mix. Since there is no gauge symmetry to fix the counterterms, we choose to quantize in such a way as to preserve holomorphic factorization. Then the computation of the fermion integrals amounts to calculating the functional determinants of the chiral differential operators. These determinants can be evaluated by the Leutwyler method [32], and in this method the only interaction between $A$ and $\bar{A}$ is given by the last term in eq. (5.55). We make the following change of variables

$$
\begin{gathered}
\bar{A} \rightarrow \bar{\partial} g_{R} g_{R}^{-1}, \\
A \rightarrow g_{L}^{-1} \partial g_{L} .
\end{gathered}
$$

and the corresponding Jacobian can be again computed according to the Leutwyler method. This Jacobian is the product of the "ghost" partition function

$$
Z_{\text {ghost }}=\int \mathcal{D} b \mathcal{D} \bar{b} \mathcal{D} c \mathcal{D} \bar{c} \exp \left[-\int d^{2} z(b \bar{\partial} c+\bar{b} \partial \bar{c})\right],
$$


where $b, c, \bar{b}, \bar{c}$ are Grassmann odd auxiliary fields in the adjoint representation of $\mathcal{G}$, and the partition function $Z_{B}\left(-N-2 c_{V}, S\right)$ of a system of two interacting WZNW models with action (2.3) and levels $k_{1}=k_{2}=\left(-N-2 c_{V}\right)$. The coupling matrix $S$ is related to the coupling matrix $G$. However, the explicit form of this relation depends on the regularization scheme and corresponds to the possibility of adding a local $A \bar{A}$ counterterm. With a chiral regularization, the relation between $S$ and $G$ is given by

$$
S=-\frac{G^{-1}}{\left(N+2 c_{V}\right)^{2}} .
$$

We finally arrive at the following relation

$$
Z_{F}(G)=Z_{F}(0) Z_{B}\left(-N-2 c_{V}, S\right) Z_{\text {ghost }},
$$

This implies that conformal points of the system of two interacting WZNW models are conformal points of the fermionic Thirring model. The fact that the system of two interacting WZNW models had $\hat{G}_{1}^{L} \times \hat{G}_{1}^{R} \times \hat{G}_{2}^{L} \times \hat{G}_{2}^{R}$ affine symmetry implies that the Thirring model should also, and so should be integrable. In the right hand side of eq. (5.61) we have the partition function of interacting WZNW models at negative level $\left(-N-2 c_{V}\right)$. For the bosonic system of two interacting WZNW models we found a nontrivial conformal point $S_{-}^{*}$ given by eq. (4.51) for large $|k|$. Since $k=\left(-N-2 c_{V}\right)$, we will consider the large $N$ limit. Note that the value of $S_{-}^{*}$ does not depend on the regularization procedure, so that the theory at the critical point is determined unambiguously. From formula (5.60), the point $S=0$ corresponds to $G=\infty$. Hence, the expansion in $S$ or $\sigma$ around free WZNW models amounts to a strong coupling expansion of the fermionic Thirring model around $G=\infty$. Of course, this would cause many problems in the fermionic theory, while in the bosonic system this is a weak coupling regime that can be studied perturbatively.

In the large $N$ limit, $S_{B}\left(-N-2 c_{V}, S\right)$ is the model (2.3) with $k \sim-N$, which can then be re-expressed as (3.24). The $g_{1}$ dependence is, as we have seen, described by a model which has an infra-red fixed point at $S_{-}^{*}$ at which the theory is a WZNW model of level given (for large $N$ ) approximately by $+N$. We then find

$$
S\left(g_{1}, g_{2}, S^{*}\right)=S_{W Z N W}\left(g_{1}, N\right)+S_{W Z N W}\left(g_{2},-N\right) .
$$


As a result, the relation (5.61) takes the form

$$
Z_{F}\left(G^{*}\right)=Z_{F}(0) Z_{W Z N W}(N) Z_{W Z N W}(-N) Z_{\text {ghost }}
$$

in the large $N$ limit.

The partition function of the $G / G$ coset is given by

$$
Z_{G / G}=Z_{W Z N W}(k) Z_{W Z N W}\left(-k-2 c_{V}\right) Z_{g h o s t},
$$

where $k$ is the level of the WZNW model on $G$ [33]. With $k=N, Z_{W Z N W}\left(-N-2 c_{V}\right) \sim$ $Z_{W Z N W}(-N)$ in the large $N$ limit, so that (5.63) takes the following form

$$
Z_{F}\left(G^{*}\right)=Z_{F}(0) Z_{G / G}
$$

in the large $N$ limit. Although $Z_{G / G}$ represents states of both negative norm and positive norm, it corresponds to a topological field theory [33] and so can be quantized in such a way that, at this conformal point, the spectrum consists of only ground states, so that the only dynamical degrees of freedom of the full theory at this critical point are described by $Z_{F}(0)$, which is the partition function of the system of free fermions. At this conformal point the (scheme-dependent) value of the Thirring coupling constant is given by

$$
G^{*}=-\frac{\left(S^{*}\right)^{-1}}{N^{2}}=-\frac{\sqrt{2}}{N}
$$

to lowest order in $1 / N$. Although the critical value of the Thirring coupling depends on the regularization procedure, the effective CFT is known exactly to leading order in $1 / N$, so that the fermionic non-Abelian Thirring model is effectively a theory of free fermions at this new conformal point. The conformal point found by Dashen and Frishman [22] also gave a free fermion theory, suggesting that the conformal point we have found is precisely the Dashen-Frishman one. Dashen and Frishman made use of the nonperturbative currentcurrent method for analysis of the conformal symmetry of the Thirring model, enabling them to find the critical value of $G$ exactly, with the result

$$
G^{*}=\frac{1}{N+c_{V}(G)}
$$

Using this result, we find the finite shift of the level along the flow from the non unitary to unitary WZNW models: $S_{W Z N W}\left(g,-N-2 c_{V}(G)\right) \rightarrow S_{W Z N W}(g, N)$. Another interesting 
observation is that the classical level $-N-2 c_{V}(G)$ of the affine currents $\mathcal{J}_{2}^{a}$ and $\overline{\mathcal{J}}_{1}^{\bar{a}}$ in eqs. (2.16) gets renormalized at the Dashen-Frishman conformal point, becoming equal to $N$.

Note that one can always add the counterterm $A \bar{A}$ with an appropriate coefficient to fix the constant $G^{*}$ at the Dashen-Frishman value. The advantage of the path integral formalism compared to the Hamiltonian current-current quantization is that the former allows to derive the effective CFT exactly. In the Dashen-Frishman approach free fermions emerge through the operator bosonization procedure.

Note that the same effect has been known for a long time in the sine-Gordon model. Coleman has proven that the sine-Gordon model is effectively a theory of free Dirac spinors at the special value of the coupling $\beta$ [34]. The sine-Gordon model in its turn can be described as the fermionic Thirring model of $S U(2)$ spinor fields with one flavour [31]. Therefore, the free fermion regime of the sine-Gordon model results in the free fermion regime of the fermionic non-Abelian Thirring model at particular values of the Thirring coupling constants. We have shown that the free fermion phase is a characteristic feature of all fermionic non-Abelian Thirring models.

Even away from the conformal points, the partition function for the fermionic nonAbelian Thirring model, which is clearly unitary, takes the factorised form

$$
Z_{F}(G)=Z_{F}(0) Z_{W Z N W}\left(-N-2 c_{V}(G)\right) Z_{\text {ghost }} Z(S)
$$

where $Z(S)$ is the partition function of the theory given by eq. (3.25). The first three factors in eq. (5.68) correspond to the partition function of free spinors coupled to gauge fields, so that their product gives a unitary partition function. Thus the factor $Z(S)$ must be the partition function of a unitary theory by itself .

\section{Conclusion}

In this paper we have found a nontrivial quantum field theory described by the action in eq. (3.25) which possesses nontrivial conformal points. We have shown that conformal points of this bosonic theory are related to critical points of the fermionic non-Abelian Thirring model. We have studied in detail one conformal point of the Thirring model at which 
the theory turns out to correspond to an effective theory of free fermions. There may be other conformal points in these models, corresponding to the many relevant perturbation operators $O^{L}$ constructed from different $L$ 's. Indeed, the models considered here might be useful in the search for a Lagrangian formulation of the affine-Virasoro construction [36.

Finally, we return to the apparent paradox in the relation between the Gross-Neveu model and the Dashen-Frishman model. Gross and Neveu quantized the Thirring model in the weak coupling regime, perturbing about the free fermion theory in terms of a small coupling $G$. We have shown that the Dashen-Frishman conformal point is to be found in a strong coupling regime of the Thirring model, expanding about $G=\infty$ (for a qualitative discussion of the given point see [23]). Although there is no well defined expansion in the strong coupling for the fermionic theory, we found a dual bosonic formulation in which the Dashen-Frishman point occurs at weak coupling. Although in the limit $N \rightarrow \infty$ the value of the Dashen-Frishman conformal point is close to $G=0$, this point can be reached only from $G=\infty$ and not from $G=0$. In fact, the $1 / N$ expansion around the free fermion model breaks down when one approches the Dashen-Frishman conformal point from the weak coupling regime. There are correlation functions which become singular at the critical value of the coupling constant [35], despite the fact that the Gross-Neveu beta function is regular at this point.There is a strong similarity between the Dashen-Frishman conformal point and the Polyakov-Wiegmann conformal point. This indicates that at the Dashen-Frishman conformal point the fermionic Thirring model undergoes a phase transition. Since Gross and Neveu have used the free fermion theory as the vacuum, their computations are only valid in the weak-coupling phase around the free fermion theory. Therefore, there is no way to see the Dashen-Frishman critical point within the GrossNeveu perturbation theory; to find it, non-perturbative or strong-coupling methods are required.

Acknowledgements:

We would like to thank Michael Green and Jonathan Evans for helpful discussions. O.S. would like to thank the PPARC for financial support.

\section{Appendix A}


In this appendix, we give a check on the concistency of our computations. Consider the following functional integral

$$
F=\int \mathcal{D} B \mathcal{D} \bar{B} \exp \left(\frac{1}{\pi} \int d^{2} z B M \bar{B}\right),
$$

where $B, \bar{B}$ are vector fields in the adjoint representation of a group $G ; M$ is a nondegenerate constant matrix. The functional integral $F$ does not contribute to the conformal anomaly at any value of $M$, and this will play a crucial role in our check.

We make the following change of variables:

$$
B=g_{1}^{-1} \partial g_{1}, \quad \bar{B}=\bar{\partial} g_{2} g_{2}^{-1},
$$

where $g_{1}, g_{2}$ are $G$-valued fields. The Jacobians are found in the same way as those for (5.58), with the result that $F$ is now given by

$$
F=Z_{B}\left(-2 c_{V}(G), \frac{M}{4 c_{V}^{2}(G)}\right) Z_{\text {ghost }},
$$

where $Z_{B}$ is the partition function of the system of two interacting level $\left(-2 c_{V}\right)$ WZNW models with the coupling matrix $M / 4 c_{V}^{2} ; Z_{\text {ghost }}$ is the partition function of the ghost-like fields (see eq. (5.59)).

Thus, the conformal properties of $F$ are determined by the conformal properties of $Z_{B}$ at level $\left(-2 c_{V}\right)$. The claim that $F$ does not contribute to the conformal anomaly amounts to the statement that the sum of the Virasoro central charges for the conformal models on the right hand side of eq. (A.3) vanishes for all $M$.This is difficult to verify explicitly in general, but can be checked at the conformal points we have found in this paper.

Let us first check the Polyakov-Wiegmann conformal point which corresponds to

$$
M_{P W}=-2 c_{V} I .
$$

At this point the theory acquires additional gauge symmetry (see section 1 ). This symmetry is sufficient to gauge away one of the two group elements. Therefore, at the PW critical point, the quantity $F$ is given by

$$
F\left(M_{P W}\right)=Z_{W Z N W}\left(-2 c_{V}\right) Z_{\text {ghost }} .
$$


It is easy to see that the Virasoro central charge in the right hand side of eq. (A.5) indeed vanishes:

$$
c=c_{W Z N W}\left(-2 c_{V}\right)+c_{g h o s t}=\frac{\left(-2 c_{V}\right) \operatorname{dim} G}{-2 c_{V}+c_{V}}-2 \operatorname{dim} G=0 .
$$

Now let us turn to the isoscalar Dashen-Frishman conformal point which corresponds to

$$
M_{D F}=2 \sqrt{2} c_{V} I
$$

According to our computations, at the DF fixed point the quantity $F$ is given by

$$
F\left(M_{D F}\right)=Z_{G / G}
$$

Obviously, the Virasoro central charge in the right hand side of eq. (A.8) vanishes.

Thus, we have proven that $F(M)$ does not contribute into the conformal anomaly at least for two different values of $M$, checking the correctness of our computations. This check leads us to suggest that for arbitray $M$ the formula for the quantity $F$ is given by

$$
F(M)=Z_{W Z N W}\left(-2 c_{V}(G)\right) Z_{\text {ghost }}
$$

This can be justified by nonperturbative computations at general Dashen-Frishman conformal points 21.

\section{Appendix B}

In this appendix, we briefly give a first order derivation of the symmetry (2.5) of the action (2.3) [29]. First, we introduce auxiliary Lie-algebra-valued variables $Q, \bar{P}$ and write the interacting action in the form

$$
\begin{aligned}
S\left(g_{1}, g_{2}, Q, \bar{P}\right) & =S_{W Z N W}\left(g_{1}, k_{1}\right)+S_{W Z N W}\left(g_{2}, k_{2}\right) \\
& -\frac{1}{2 \pi} \int d^{2} z \operatorname{Tr}\left(k_{1} g_{1}^{-1} \partial g_{1} \bar{P}+k_{2} Q \bar{\partial} g_{2} g_{2}^{1}-\frac{1}{2} Q \cdot S^{-1} \cdot \bar{P}\right) .
\end{aligned}
$$

If we eliminate $Q, \bar{P}$ via their equations of motion, we regain (2.3). Instead, we express $Q, \bar{P}$ in terms of new variables $h_{1}, h_{2}$ as

$$
\bar{\partial} h_{1} h_{1}^{-1}=\bar{P}, \quad h_{2}^{-1} \partial h_{2}=Q
$$


and use the Polyakov-Wiegmann formula to obtain

$$
\begin{aligned}
S\left(g_{1}, g_{2}, Q, \bar{P}\right) & =S_{W Z N W}\left(g_{1} h_{1}, k_{1}\right)+S_{W Z N W}\left(h_{2} g_{2}, k_{2}\right) \\
& -S_{W Z N W}\left(h_{1}, k_{1}\right)-S_{W Z N W}\left(h_{2}, k_{2}\right)+\frac{1}{4 \pi} \int d^{2} z \operatorname{Tr}^{2} Q \cdot S^{-1} \cdot \bar{P} .
\end{aligned}
$$

This is invariant under the transformations

$$
g_{1} \rightarrow \bar{\Omega}_{1}(\bar{z}) g_{1} h_{1} \Omega_{1}(z) h_{1}^{-1}, \quad g_{2} \rightarrow h_{2}^{-1} \bar{\Omega}_{2}(\bar{z}) h_{2} g_{2} \Omega_{2}(z)
$$

as well as

$$
h_{1} \rightarrow h_{1} \Lambda_{1}(z), \quad h_{2} \rightarrow \bar{\Lambda}_{2}(\bar{z}) h_{2} .
$$

The original model is regained on imposing

$$
\bar{\partial} h_{1} h_{1}^{-1}=2 k_{2} \operatorname{Tr} S \bar{\partial} g_{2} g_{2}^{-1}, \quad h_{2}^{-1} \partial h_{2}=2 k_{1} \operatorname{Tr} S g_{1}^{-1} \partial g_{1}
$$

\section{Appendix C}

In this appendix, we shall discuss further the OPE given in eq. (4.45). The relevant operator $O^{L, \bar{L}}$ is the descendant at the first affine level of the adjoint-adjoint primary operator $\phi^{a \bar{a}}$. Let us compute first the OPE of $\phi^{a \bar{a}}$. For simplicity, we will consider the special case $G=S U(2)$. Because of the holomorphic factorization, we can present $\phi^{a \bar{a}}(z, \bar{z})$ as $\phi^{a}(z) \bar{\phi}^{\bar{a}}(\bar{z})$. Then we can proceed with the left (holomorphic) part of $\phi^{a \bar{a}}$,

forgetting about the right (antiholomorphic) part. Similar results can be derived for the right (antiholomorphic) part. Then

$$
\phi^{a}(z) \phi^{b}(0)=\frac{[j=0]}{z^{2 \Delta_{\phi}}}+\frac{C_{c}^{a b} \phi^{c}(0)}{z^{\Delta_{\phi}}}+\frac{[j=2]}{z^{2 \Delta_{\phi}-\Delta_{2}}}+\ldots
$$

Here $[j]$ are affine primaries with spin $j$ and conformal dimensions

$$
\Delta_{j}=\frac{j(j+1)}{k+2}, \quad \Delta_{\phi}=\Delta_{1}
$$

$C_{c}^{a b}$ are structure constants.

For all positive $k, \Delta_{\phi}-\Delta_{j}<0$, when $j>1$. Therefore, all higher spin representations with $j \geq 2$ do not contribute to the singular part of the OPE in eq. (C.1) 37. However, 
we are interested in $k<-2 c_{V}$, that is for $S U(2), k<-4$. Hence, in this case all higher spin primaries can appear in the singular part of the OPE. Thus, it may seem as if all these singularities could appear in the OPE of the operator $O^{L}$ with itsel fmodifying the formula (4.45). For example, dimensional analysis permits an operator of spin 2 on the right handside of (4.45), so that

$$
O^{L}(z) O^{L}(0) \sim \frac{C_{[2]}[2]}{z^{2+2 \Delta_{\phi}-\Delta_{2}}}+\ldots
$$

for some structure constant $C_{[2]}$.

Now we want to prove that the only singular terms which may appear in the OPE of two operators $O^{L}$ are given by eq. (4.45), so that in particular $C_{[2]}=0$. Suppose that eq. (C.3) is correct. Acting with $J_{0}^{a}$ on both sides of (C.3) gives

$$
\left[J_{0}^{a}, O^{L}(z)\right]\left|O^{L}(0)\right\rangle+O^{L}(z) J_{0}^{a}\left|O^{L}(0)\right\rangle=\frac{C_{[2]} J_{0}^{a}|[2]\rangle}{z^{2+2 \Delta_{\phi}-\Delta_{2}}}+\ldots
$$

We have also acted on the $S L(2, C)$ vacuum, so that we deal with states, not operators.

Let us consider the case

$$
L_{a b} \sim g_{a b}
$$

This matrix, for example, arises in eq. (4.46). Then it is easy to show that the left hand side of eq. (C.4) vanishes, since

$$
J_{0}^{a} J_{-1}^{b}\left|\phi^{b}\right\rangle=0
$$

We have used the relation

$$
\left[J_{n}^{a}, \phi^{b}(z)\right]=\oint \frac{d w}{2 \pi i} w^{n} J^{a}(w) \phi^{b}(z)=z^{n} f_{c}^{a b} \phi^{c}(z)
$$

This then implies the following condition

$$
C_{[2]} J_{0}^{a}|[2]\rangle=0
$$

Acting with $J_{0}^{a}$ and contracting indices, we obtain

$$
C_{[2]} g_{a b} J_{0}^{a} J_{0}^{b}|[2]\rangle=0,
$$

but since $[j]$ has spin $j$, we also have

$$
g_{a b} J_{0}^{a} J_{0}^{b}|[j]\rangle=j(j+1)|[j]\rangle
$$


which is non zero. Thus, in order for eq. (C.8) to be satisfied, the coefficient $C_{[2]}$ must be zero,

$$
C_{[2]}=0 .
$$

Following the same chain of arguments, one can prove also that

$$
C_{[j>2]}=0
$$

This proves the OPE given by eq. (4.45) for the matrix $L$ in eq. (C.5).

This result can be generalized to arbitrary matrices $L$ and arbitrary groups $G$ using the arguments given in section 4 and [28], but the above constitutes an explicit check for the special case $G=S U(2), L_{a b} \sim g_{a b}$.

\section{References}

[1] D. Karabali, Q-H. Park, H. J. Schnitzer and Z. Yang, Phys. Lett. B216 (1989) 307; D. Karabali and H. J. Schnitzer, Nucl. Phys. B329 (1990) 649.

[2] S. Hwang and H. Rhedin, Nucl. Phys. B406 (1993) 165.

[3] A. A. Belavin, A. M. Polyakov and A. B. Zamolodchikov, Nucl. Phys. B241 (1984) 333.

[4] D. Friedan, Z. Qiu and S. Shenker, Phys. Rev. Lett. 52 (1984) 1575.

[5] E. Witten, Phys. Rev. D44 (1991) 175.

[6] A. A. Tseytlin, Conformal sigma models corresponding to gauged Wess-ZuminoWitten theories, Preprint CERN-TH. 6804/93, hep-th/9302083.

[7] K. Bardakci, M. Crescimanno and E. Rabinovici, Nucl. Phys. B344 (1990) 344.

[8] S. P. Novikov, Usp. Math. Nauk 37 (1982) 3.

[9] E. Witten, Commun. Math. Phys. 92 (1984) 455.

[10] V. G. Knizhnik and A. B. Zamolodchikov, Nucl. Phys. B247 (1984) 83. 
[11] A. M. Polyakov and P. B. Wiegmann, Phys. Lett. 141B (1984) 223.

[12] B. Rai and V. G. J. Rodgers, Nucl. Phys. B341 (1990) 119.

[13] S. Bellucci, D. A. Depireux and S. J. Gates Jr., Phys. Lett. B232 (1989) 67.

[14] D. A. Depireux, S. J. Gates Jr. and Q-H. Park, Phys. Lett. B224 (1989) 364.

[15] S. J. Gates Jr., S. V. Ketov, S. M. Kuzenko and O. A. Soloviev, Nucl. Phys. B362 (1991) 199.

[16] D. Gonzales and A. N. Redlich, Phys. Lett. 147B (1984) 150.

[17] A.N. Redlich and H. J. Schnitzer, Phys. Lett. 167B (1986) 315.

[18] D. Karabali, Q-H. Park and H. J. Schnitzer, Phys. Lett. B205 (1988) 267; Nucl. Phys. B323 (1989) 572.

[19] A. N. Theron, F. A. Schaposhnik, F. G. Scholtz and H. B. Geyer, Non-Abelian bosonization from factored coset models in path integrals, Preprint (Oct, 1994).

[20] O. A. Soloviev, Conformal non-Abelian Thirring models, QMW-TH 93-19, hepth/9307163.

[21] O. A. Soloviev, Mod. Phys. Lett. A8 (1993) 3071.

[22] R. Dashen and Y. Frishman, Phys. Lett. B46 (1973) 439; Phys. Rev. D11 (1974) 2781.

[23] P. K. Mitter and P. H. Weisz, Phys. Rev. D8 (1973) 4410.

[24] D. J. Gross and A. Neveu, Phys. Rev. D10 (1974) 3235.

[25] K. Bardakci, Nucl. Phys. B431 (1994) 191

[26] O. A. Soloviev, Phys. Lett. B321 (1994) 365.

[27] O. A. Soloviev, Nucl. Phys. B431 (1994) 206.

[28] O. A. Soloviev, Nucl. Phys. B440 (1995) 373. 
[29] O. A. Soloviev, Phys. Lett. B309 (1993) 275.

[30] E. Witten, Nucl. Phys. B371 (1992) 191.

[31] S. J. Gates Jr. and O. A. Soloviev, Phys. Lett. B294 (1992) 342.

[32] H. Leutwyler, Phys. Lett. B152 (1985) 78; B153 (1985) 65; Helv. Phys. Acta 59 (1986) 201.

[33] M. Spiegelglas and S. Yankielowicz, Nucl. Phys. B393 (1993) 301; O. Aharony, O. Gano, J. Sonnenschein, S. Yankielowicz, Nucl. Phys. B399 (1993) 527.

[34] A. Coleman, Phys. Rev. D11 (1975) 2088.

[35] D. Kutasov, Phys. Lett. B227 (1989) 68.

[36] M. B. Halpern and E. Kiritsis, Mod. Phys. Lett. A4 (1989) 1373; A4 (1989) 1797 (E); A. Yu. Morozov, A. M. Perelomov, A. A. Rosly, M. A. Shifman and A. V. Turbiner, Int. J. Mod. Phys. A5 (1990) 803.

[37] A. B. Zamolodchikov and V. A. Fateev, Sov. J. Nucl. Phys. 43 (1986) 657. 